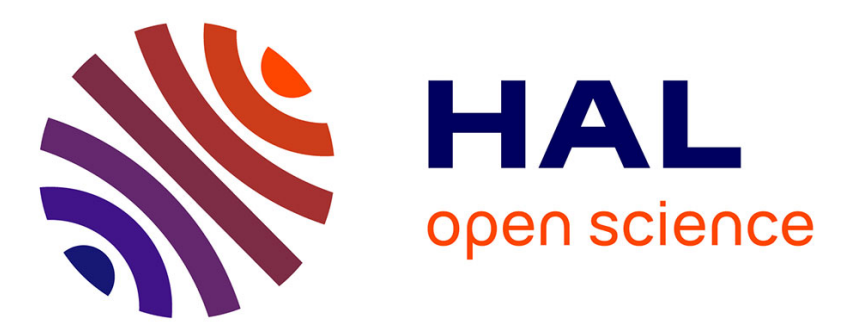

\title{
Compared prediction of the experimental failure of a thin fibrous tissue by two macroscopic damage models
}

Aline Bel-Brunon, Michel Coret, Karine Bruyere-Garnier, Alain Combescure

\section{To cite this version:}

Aline Bel-Brunon, Michel Coret, Karine Bruyere-Garnier, Alain Combescure. Compared prediction of the experimental failure of a thin fibrous tissue by two macroscopic damage models. Journal of the mechanical behavior of biomedical materials, 2013, 27, pp. 262-272. 10.1016/j.jmbbm.2013.05.019 . hal-00919691

\section{HAL Id: hal-00919691 https://hal.science/hal-00919691}

Submitted on 18 Mar 2019

HAL is a multi-disciplinary open access archive for the deposit and dissemination of scientific research documents, whether they are published or not. The documents may come from teaching and research institutions in France or abroad, or from public or private research centers.
L'archive ouverte pluridisciplinaire HAL, est destinée au dépôt et à la diffusion de documents scientifiques de niveau recherche, publiés ou non, émanant des établissements d'enseignement et de recherche français ou étrangers, des laboratoires publics ou privés. 


\title{
Compared prediction of the experimental failure of a thin fibrous tissue by two macroscopic damage models.
}

\author{
A. Bel-Brunon ${ }^{\mathrm{a}, \mathrm{b}, \mathrm{c}, *}$, M. Coret ${ }^{\mathrm{a}, \mathrm{b}}, \mathrm{K}$. Bruyère-Garnier ${ }^{\mathrm{a}, \mathrm{c}}, \mathrm{A}$. Combescure Com $^{\mathrm{a}, \mathrm{b}}$ \\ ${ }^{a}$ Université de Lyon, Lyon, F-69000, France \\ ${ }^{b}$ INSA-Lyon, LaMCoS UMR5259, F-69621, France \\ cIfsttar, UMRT9406, LBMC, F-69675, Bron, France - Université Lyon 1, F-69622, Villeurbanne, France
}

\begin{abstract}
Several models for fibrous biological tissues have been proposed in the past, taking into account the fibrous microstructure through different homogenization methods. The aim of this paper is to compare theoretically and experimentally two existing homogenization methods - the Angular Integration method and the Generalized Structure Tensor method - by adapting them to a damage model for a planar fibrous tissue made of linear elastic and brittle fibers. The theoretical implementation of the homogenization methods reveals some differences once damage starts in the fibrous tissue; in particular, the anisotropy of the tissue evolves differently. The experimental aspect of this work consists in identifying the parameters of the damage model, with both homogenization methods, using inflation tests until rupture on a biological membrane. The numerical identification method is based on the simulation of the tests with the real geometry of the samples and the real boundary conditions computed by Stereo Digital Image Correlation. The identification method is applied to human liver capsule. The collagen fibers Young's modulus (19 $\pm 6 \mathrm{MPa}$ ) as well as their ultimate longitudinal strain $(33 \pm 4 \%)$ are determined; no significant difference was observed between the two methods. However, by using the experimental boundary conditions, we could observe that the damage progression is faster for the Angular Integration version of the model.
\end{abstract}

Keywords: damage model, homogenization, fibrous tissue, numerical identification, Stereo Digital Image Correlation, human liver capsule

\section{Introduction}

In the field of biomechanics of soft tissues, a lot of studies have been focused on the characterization of the behavior of biological tissues and organs. This is due to the numerous medical applications of a human body model, which usually remain in the physiological range of loadings. However, the potentialities of a virtual human body including information about failure of the tissues are important in several fields, including road safety and surgery. Many fatal cases caused by car crashes and reported in the literature are due to abdominal organ injuries, especially the spleen, the liver and the kidney (Tinkoff et al, 2008). Predicting the occurrence of abdominal injuries by car crash simulation would improve user safety by suggesting technical changes in the

${ }^{*}$ Corresponding author: Lehrstuhl für Numerische Mechanik, Boltzmannstrasse 15, D-85747 Garching b. München, Germany. Email: bel@lnm.mw.tum.de. passive and active safety systems. Besides, a difficulty in abdominal surgery is to handle the organs without damaging them. Using surgical simulation to predict the overloads responsible for injuries could be useful to prevent them. For these applications, a constitutive law representing the elastic or viscoelastic behavior of a soft tissue, associated to geometrical data - e.g. external shape - and interaction data - e.g. contact behavior with neighboring organs - is not sufficient to predict injuries; a human body model also requires failure properties for these biological tissues.

Two main issues are associated with the study of soft biological tissues failure properties: advanced experimental methods are needed to guarantee the good quality of the measurements despite the softness and the living aspect of the tissues; sophisticated models are required to represent the failure mechanisms occurring in these complex and structured materials. These two features also need to be coupled so that the model complexity (number of parameters) and the experimental possi- 
bilities (imaging, identification) are consistent.

Investigating failure properties of soft tissues is experimentally challenging as failure is a local and unstable phenomenon that can be highly influenced by the experimental conditions. The use of full-field measurements for studying failure has been shown (Brunon et al, 2010) and is particularly adapted to soft tissues, as the boundary conditions of the experimental tests are more difficult to control and thus to repeat from one sample to the other. This is due for instance to the existence of multiple stress-free states for a soft tissue, the compression of the tissue in the clamps, the difficulty to cut samples of the same shape, etc. Also, using inflation tests to characterize biological membranes can help making 10 the tests more repeatable as the failure does not occur at 102 the sample edges, where some failure initiations can be 103 created during the cutting phase. However, this loading 104 mode is not common although it usually corresponds to 105 more realistic loadings than uniaxial tension and guar- 106 antees a better understanding of non-linearity and possi- 107 ble anisotropy of the tissue (Johannknecht and Jerrams, 108 1999). Boyce et al. and Bischoff et al. among oth- 109 ers have used this type of loading to characterize the 110 cornea and artery behavior (Boyce et al, 2008; Bischoff 111 et al, 2009). A few papers describe inflation tests on 112 biological tissues until rupture. Mohan and Melvin de- 113 termined the ultimate stress and strain of human aor- 114 tic tissue using an analytic model of inflated membrane 115 (Mohan and Melvin, 1983). Marra et al. calculated the 116 failure strength of porcine aorta from the measurements 117 of the global deformation and applied pressure (Marra 118 et al, 2006). Kim et al. determined a nonlinear consti- 119 tutive law and rupture criterion for the artery (Kim et al, 120 2011). In a previous paper (Brunon et al, 2011), we de- ${ }_{121}$ termined the hyperelastic constitutive law and ultimate ${ }_{122}$ strain of the liver capsule. However none of these stud- ${ }_{123}$ ies consisted in the identification of a damage model. ${ }_{124}$

In terms of modeling, several models for fibrous tis- 125 sues are available in the literature. They consider the ${ }_{126}$ fibrous microstructure to drive the macroscopic behav- ${ }_{127}$ ior of the tissue. Either the distribution of the fibers ${ }_{128}$ and their reference state are described statistically and ${ }_{129}$ further identified (Lanir, 1983; Decraemer et al, 1980); 130 or some histological evidences lead to the construc- 131 tion of specific representations for the microstructure, ${ }_{132}$ such as the structure tensor proposed in (Gasser et al, 133 2006). These two types of models correspond to two 134 main homogenization methods, respectively an Angu- 135 lar Integration (AI) method and a Generalized Structure ${ }_{136}$ Tensor (GST) method. Several physical phenomena ${ }_{137}$ such as viscoelasticity, plasticity, growth and remod- 138 eling are also considered (Gasser et al, 2002; Gleason ${ }_{139}$

\section{et}

\section{3 ory} lent strain over the past history. In (Rodriguez et al, 2006) however, the damage in the fiber bundles is controlled by a probability density function that reflects the stochastic waviness of the fibers in their reference state; it is therefore better suited to biological soft tissues as collagen fibers are usually wavy in an unloaded biological tissue, see e.g. (Viidik, 1972; Orberg et al, 1982; Hill et al, 2012).

In this study, we focus on two homogenization methods proposed in the literature and investigate their differences in the range of damaging loads. The AI method proposed by (Lanir, 1983) and the GST method proposed by (Gasser et al, 2006) have been theoretically compared in (Cortes et al, 2010) for physiological ranges of loading, i.e. without any damage. Limits of the GST have been emphasized for fibrous distributions close to isotropy, but the differences between AI and GST methods vanish in the case of quasi-equibiaxial loading. In the present work, the experimental test case combines isotropic tissue and quasi-equibiaxial loading. The experiments are mainly devoted to provide data for the failure mechanism of this kind of tissue. But a byproduct of these tests is also to produce some experimental data which allow comparing the non linear response of the two models in such a configuration.

Although several sophisticated models are available in the literature to account for various physical features - viscoelasticity e.g. (Limbert et al, 2004), anisotropy e.g. (Ateshian, 2007), fiber crimp e.g. (Cacho et al, 2007), etc - we chose to compare the homogenization methods using a simple model describing an isotropic fibrous membrane, made of linear elastic brittle fibers and loaded with biaxial tension. The tissue macroscopic damage is due to fiber rupture at the microscale. This is the focus of the second part of the paper. A method to identify the two versions of the obtained damage model using inflation tests and full-field measurements is then presented in the third part. The fourth part is an application of this method on human liver capsule; results are discussed in the fifth part. 


\section{Construction of the damage model and theoreti-} cal comparison of the homogenization methods

The proposed model consists of a damage model for the tissue that is homogenized with two homogenization methods. Some simplifying assumptions (negligible reorientation of the fibers, linear elastic behavior of the fibers or the tissue) of this academic model help making the framework as clear as possible to focus on the two main points that are the comparison of the homogenization methods and the identification method.

\subsection{General framework}

This section takes up the general framework of 189 (Gasser et al, 2006).

We consider a plane tissue consisting of a groundma- 191 trix and fibers. We consider an additive decomposition 192 of the Helmoltz free-energy function $\psi$, defined per unit 193 reference volume, into the free energy of the groundma- 194 trix $\psi_{m}$ and the free energy of the fibers $\psi_{f}$ :

$$
\psi=\psi_{m}+\psi_{f}
$$

For the sake of clarity, the matrix contribution, al- 200 ready assessed in (Gasser et al, 2006) is not described 201 here. Only the fibers contribution is detailed through 202 two homogenization methods.

\subsection{Description of the two homogenization methods}

Let us consider a fibrous membrane made of linear elastic brittle fibers and suppose that we know the influence of a biaxial tension loading on the fibers fracture. We can proceed to homogenize the behavior. The most 205 commonly used homogenization methods are the ones 206 described in (Lanir, 1983) - AI method - and (Gasser et al, 2006) - GST method. The GST method has been shown to have some limitiations (Cortes et al, 2010). It is nevertheless reasonable for a quasi-equibiaxial strain state which is the case of this study. It will be extended by adding a fiber fracture model in this paper. The same 207 extension will be proposed for the AI model.

\subsubsection{The concept of fiber density function}

In order to describe the strain energy in the tissue, we need to introduce the concept of angular fiber density, denoted $\rho(\xi)$. This function defines the fraction of fibers whose orientation belongs to the interval $[\xi, \xi+d \xi]$. Before damage, this function is considered to be normalized, i.e.:

$$
\frac{1}{\pi} \int_{-\frac{\pi}{2}}^{\frac{\pi}{2}} \rho(\xi) \mathrm{d} \xi=\frac{1}{\pi} \int_{A_{0}} \rho(\xi) \mathrm{d} \xi=1
$$

In the case of a homogeneous distribution, i.e. $\rho(\xi)=$ constant, one has $\rho(\xi)=1 \forall \xi \in A_{0}=[-\pi / 2, \pi / 2]$. Once damage starts, the density is a function of the damage state $\mathbf{D}$. In the present work, $\mathbf{D}$ defines the range of angles of the undamaged fibers i.e. where $\rho(\xi)$ is not null.

\subsubsection{The Angular Integration (AI) homogenization method}

In this method, the free energy of the fibrous part of the tissue is assumed to be the integral of the contribution of the strained, but undamaged, fibers. A fiber subjected to a Green-Lagrange strain field $\mathbf{E}$ is strained only along its longitudinal axis $\mathbf{n}(\xi)$ and its strain energy is $\phi_{f}=\phi_{f}\left(\varepsilon_{f}\right)$ i.e. $\phi_{f}(\xi, \mathbf{E})$, whose expression depends on the constitutive equation of the fiber. $\mathbf{n}$ is the unit vector associated to the initial orientation of the fiber. As described in the next section, we neglect the change of orientation between the fibers and the local reference frame during loading. Hence the vector $\mathbf{n}$ which represents the direction of each fiber with respect to the continuous material frame does not change during loading. Therefore, on the tissue's scale, the free energy $\psi_{f}^{A I}$ of the fibers is

$$
\psi_{f}^{A I}(\mathbf{E}, \mathbf{D})=\frac{1}{\pi} \int_{A_{0}} \rho(\xi, \mathbf{D}) \phi_{f}(\xi, \mathbf{E}) \mathrm{d} \xi
$$

The expression of the second Piola-Kirchhoff stress tensor (PK2) is:

$$
\mathbf{S}_{f}^{A I}=\frac{\partial \psi_{f}^{A I}(\mathbf{E}, \mathbf{D})}{\partial \mathbf{E}}=\frac{1}{\pi} \int_{A_{0}} \rho(\xi, \mathbf{D}) \frac{\partial \phi_{f}(\xi, \mathbf{E})}{\partial \mathbf{E}} \mathrm{d} \xi
$$

Now let us consider the simplified case where the fibers behave linearly before damage. This assumption is strong as we know that the behavior of a collagen fiber cannot be considered linear above $10 \%$ of strain (Svensson et al, 2010). The expression of stress tensor PK2 is then:

$$
\mathbf{S}_{f}^{A I}=\frac{E}{\pi} \int_{A_{0}} \rho(\xi, \mathbf{D})(\mathbf{M}: \mathbf{E}) \mathbf{M} \mathrm{d} \xi
$$


where $\mathbf{F}$ is the deformation gradient and $J=\operatorname{det}(\mathbf{F})$ Thus:

$\mathbf{T}_{f}^{A I}=J^{-1} \mathbf{F} \cdot \mathbf{S}_{f}^{A I} \cdot \mathbf{F}^{T}=\frac{2 E}{J \pi} \mathbf{F} \cdot \int_{A_{0}} \rho(\xi, \mathbf{D})(\mathbf{M}: \mathbf{E}) \mathbf{M} \mathrm{d} \xi \cdot \mathbf{F}^{T}{ }_{245}$

where $E$ is the Young's modulus of the fiber's mate- ${ }_{236}$ rial and $\mathbf{M}=\mathbf{n}(\xi) \otimes \mathbf{n}(\xi)$ the orientation tensor. $\quad 237$

The Cauchy stress tensor is obtained using the fol- ${ }^{238}$ lowing expression:

$$
\mathbf{T}=J^{-1} \mathbf{F} . \mathbf{S} . \mathbf{F}^{T}
$$

2.2.3. The Generalized Structure Tensor (GST) ho- ${ }_{248}$ mogenization method

The GST method is derived from in (Gasser et al, 2006), among others. We introduce a generalized 250 second-order structure tensor $\mathbf{H}$ defined by Eq.8. This 251 tensor is used as a macroscopic projector of the strain tensor onto the structure of the undamaged fibers.

$$
\mathbf{H}=\frac{1}{\pi} \int_{A_{0}} \rho(\xi, \mathbf{D}) \mathbf{n}(\xi) \otimes \mathbf{n}(\xi) \mathrm{d} \xi
$$

Thus, the constitutive law is applied to the tissue ${ }^{250}$ rather than to its constituent fibers, taking the scalar 250 $E_{h}=\mathbf{H}: \mathbf{E}$ as the strain value to express the macro- ${ }_{259}$ scopic strain energy $\psi_{f}^{G S T}$. In the linear case we get:

$$
\psi_{f}^{G S T}=\frac{1}{2} E E_{h}^{2}=\frac{1}{2} E(\mathbf{H}: \mathbf{E})^{2}
$$

From that expression, we deduce the PK2 tensor cor- 265 responding to the GST model and the Cauchy stress:

$$
\begin{array}{r}
\mathbf{S}_{f}^{G S T}=\frac{\partial \psi_{f}^{G S T}}{\partial \mathbf{E}}=E(\mathbf{H}: \mathbf{E}) \mathbf{H} \\
\mathbf{T}_{f}^{G S T}=J^{-1} \mathbf{F} \cdot \mathbf{S}_{f}^{G S T} \cdot \mathbf{F}^{T}=\frac{E}{J}(\mathbf{H}: \mathbf{E}) \mathbf{F} \cdot \mathbf{H} \cdot \mathbf{F}^{T}
\end{array}
$$

\subsection{A simple model for the fibers fracture}

In this section, we propose a simple model to describe 275 the evolution of the macroscopic damage denoted $\mathbf{D}$ of 276 a planar fibrous tissue subjected to biaxial tension load- 277 ing, which is assumed to be the result of fiber breakage ${ }_{278}$ on the microscale. This section has two objectives:
- first, to build a damage evolution law to carry out a calculation on the macroscale taking into account the anisotropic nature of the damage due to the microstructure;

- second, to compare the extension to fracture of the two homogenization methods presented in the previous section.

The underlying assumptions of this section are the following:

- the fibers are rectilinear (no initial crimp), linear elastic and brittle;

- prior to damage, the angular distribution function of the fibers is known, continuous and strictly positive;

- the phenomenon of fiber reorientation during loading is neglected;

- the principal directions of the biaxial strain loading do not change.

The assumption that the distribution is strictly positive helps simplifying the framework as it leads to a simple expression of the damage angles without extra condition of existence of fibers in a specific direction. The third assumption relies on the conclusions of Sacks and Gloeckner and Liao et al. which observed that the closer the loading to equibiaxiality, the lesser the reorientation of the fibers (Sacks and Gloeckner, 1999; Liao et al, 2005). Therefore, in the present study which focuses on biaxial loading close to equibiaxiality, we shall ignore fiber reorientation. This assumption helps simplifying the framework of the model. Let us quote however that the description of the damage variables evolution that is given in the paper does not require this assumption. This model is academic and is designed to produce clear conclusions when we compare the two homogenization methods proposed in the previous section. It can be extended using a two scale approach to describe more realistic situations as uncrimping, damage fibers, non isotropic fiber orientations; if they are based on statistical distributions of properties - e.g. in (Cacho et al, 2007) for uncrimping - the price to pay to these extensions is a larger number of internal variables to describe the small scale state and therefore, an increased computational cost and a decreased identifiability of the model. 


\subsubsection{Parameterization of the problem}

Let us consider a planar fibrous tissue. In the material plane $\left(\mathbf{X}_{\mathbf{m}}, \mathbf{Y}_{\mathbf{m}}\right)$, the direction of a fiber is characterized by the angle $\xi \in[-\pi / 2, \pi / 2]$ and its initial direction vector $\mathbf{n}$ defined by:

$$
\mathbf{n}=\cos \xi \mathbf{X}_{\mathbf{m}}+\sin \xi \mathbf{Y}_{\mathbf{m}}
$$

The tissue is subjected to a biaxial strain character- ${ }_{309}$ ized by the macroscopic Green-Lagrange strain tensor ${ }_{310}$ E described in Cartesian coordinates by:

$$
\begin{aligned}
\mathbf{E} & =\varepsilon_{r} k\left(\cos \varphi \mathbf{X}_{\mathbf{m}} \otimes \mathbf{X}_{\mathbf{m}}+\sin \varphi \mathbf{Y}_{\mathbf{m}} \otimes \mathbf{Y}_{\mathbf{m}}\right) \\
& =E_{1} \mathbf{X}_{\mathbf{m}} \otimes \mathbf{X}_{\mathbf{m}}+E_{2} \mathbf{Y}_{\mathbf{m}} \otimes \mathbf{Y}_{\mathbf{m}}
\end{aligned}
$$

where $\varepsilon_{r}$ is the ultimate longitudinal strain of the ${ }^{316}$ fibers and $\varphi$ is the loading angle. From here on, we ${ }^{317}$ will assume that $\varphi \in[0, \pi / 2]$ and $k \geq 0$, which implies 318 strict biaxial tension, i.e. no compression and possibly ${ }_{319}$ different amplitude in both tension directions.

The fibers constituting the tissue are uniaxial ele- ${ }^{321}$ ments which can withstand only solicitations along their 322 axis. We define the longitudinal strain $\varepsilon_{f}$ of a fiber ori- ${ }^{323}$ ented along an angle $\xi$ by:

$$
\varepsilon_{f}=\mathbf{n}(\xi) \cdot \mathbf{E} \mathbf{n}(\xi)=k \varepsilon_{r}\left(\cos \varphi \cdot \cos ^{2} \xi+\sin \varphi \sin ^{2} \xi\right)
$$

This corresponds to the Green strain. We can observe that for $\varphi=\frac{\pi}{4}$ all the fibers are loaded equally; then, their longitudinal strain is $\varepsilon_{f}=\frac{k \varepsilon_{r}}{\sqrt{2}}$. Also, differentiating $\varepsilon_{f}$ with respect to $\xi$ shows that the most highly loaded fibers are oriented along the principal directions of the strain tensor, that is $\xi=0$ or $\xi=\frac{\pi}{2}$, see details in (BelBrunon et al, 2012).

\subsubsection{Initial elasticity range}

The elasticity range $\mathcal{D}$ of a fiber is defined in the strain space by:

$$
\mathcal{D}=\left\{\varepsilon_{f} \mid \varepsilon_{f}-\varepsilon_{r}<0\right\}
$$

The corresponding elasticity range of the tissue, denoted $\mathcal{S}$ is simply:

$$
\mathcal{S}=\left\{\mathbf{E} \mid \forall \xi, \mathbf{n}(\xi) . \mathbf{E} \cdot \mathbf{n}(\xi)-\varepsilon_{r}<0\right\}
$$

The shape of $\mathcal{S}$ corresponds to the resolution of the equation $\varepsilon_{f}-\varepsilon_{r}<0$ and is simply described by:

$$
\begin{cases}k<\frac{1}{\cos \varphi} & \forall \varphi \in\left[0, \frac{\pi}{4}\right] \\ k<\frac{1}{\sin \varphi} & \forall \varphi \in\left[\frac{\pi}{4}, \frac{\pi}{2}\right]\end{cases}
$$

At the boundary of $\mathcal{S}$, at least one fiber breaks as the non-rupture criterion is not respected anymore (Eq.16). The first fiber to break is always the one oriented along $\xi=0$ if $\varphi \leq \frac{\pi}{4}$ or the one oriented along $\xi=\frac{\pi}{2}$ if $\varphi \geq \frac{\pi}{4}$. The next section describes the damage process of the fibrous tissue.

\subsubsection{Evolution of the damage $\mathbf{D}$}

In this paper we only mention the case of a proportional loading (i.e. with $\varphi$ constant) for the sake of simplicity. More details on all cases can be found in (BelBrunon et al, 2012). Let us assume that the tissue is subjected to a proportional strain loading so as to reach a point defined by $(k, \varphi)$ out of the boundaries defined by Eq.18.

The damaged state at the microscale is then defined by two subsets: the subset of healthy fibers and the subset of broken ones. These sets are defined by two angles $\xi_{1}$ and $\xi_{2}$. The vector of the two damage variables $\xi_{1}$ and $\xi_{2}$ is denoted $\mathbf{D}$ which characterizes the damage state of the tissue. These angles are obtained by the solution of inequality $\varepsilon_{f}(\xi)-\varepsilon_{r}>0$. A proportional loading with an intensity $k$ greater than the bounds defined in Eq.18 $\varphi=\frac{\pi}{4}:$ all fibers break simultaneously at $k=\sqrt{2}($ Eq.15)

$\varphi \in\left[\frac{\pi}{4}, \frac{\pi}{2}\right]:\left\{\begin{array}{l}\text { all fibers are broken } \forall \xi \in\left[-\frac{\pi}{2},-\xi_{2}\right] \cup\left[\xi_{2}, \frac{\pi}{2}\right] \\ \xi_{2}=\arccos \sqrt{\frac{1-k \sin \varphi}{k(\cos \varphi-\sin \varphi)}}\end{array}\right.$

330 as detailed in (Bel-Brunon et al, 2012).

\subsection{Comparison of the homogenization methods}

This section compares the properties of the two homogenization methods when applied to the damage model described in the previous section. The test case 
presented here corresponds to biaxial tension with a ${ }_{358}$ greater amplitude in the 11 direction than in the $22 \mathrm{di}$ rection (Fig.1); all the following plots of this section correspond to this particular loading case which leads to a slower increase of damage than equibiaxial loading and therefore, helps understanding the damage process.

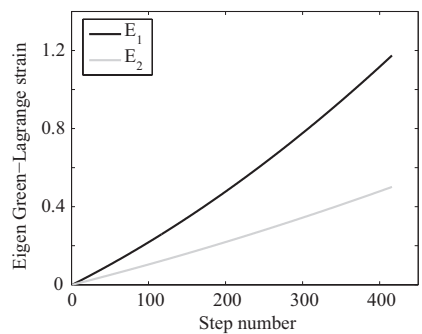

Figure 1: Components of the Green-Lagrange strain tensor of the test case.

\subsubsection{Macroscopic structure tensor properties}

An example of the evolution of the diagonal components of $\mathbf{H}$ for a loading up to rupture and for a uniform angular distribution of the fibers prior to damage, is given in Fig.2. When these two components are null, all the fibers are broken and only the groundmatrix carries the load. It can also be observed that $H_{12}=H_{21}=0$ because the function $\cos * \sin$ is odd.

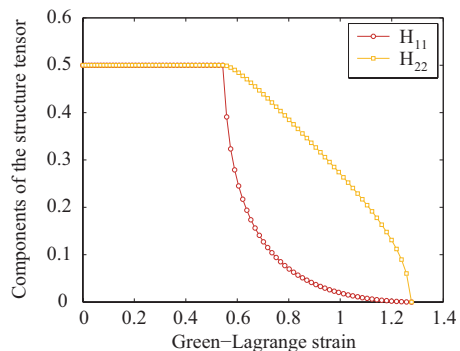

Figure 2: Evolution of the components of the structure tensor for an increasing strain amplitude.

\subsubsection{Influence of the homogenization method on the} stress-strain curves

A plot of the fibers contribution to the strain energy 376 for each homogenization method (Fig.3) shows that un- 377 der the current assumptions of uniform angular dis- 378 tribution prior to damage and brittle linear fibers, the 379 two models behave differently only when fibers start to 380 break. This difference can be observed by expanding the ${ }_{381}$ expressions of these energies (Eq.22,23). Let us denote 382
$X$ the term $\left(E_{1} \cos ^{2} \xi+E_{2} \sin ^{2} \xi\right)$. The AI fiber energy is the integral of $X^{2}$ whereas the GST one is the square of the integral of $X$. Indeed, we can observe that with $\xi_{1}$ and $\xi_{2}$ constant (especially prior to damage), the ratio of $\psi_{f}^{G S T}$ to $\psi_{f}^{A I}$ is constant throughout the loading and independent of the value of the elastic parameter $E$.

$\psi_{f}^{A I}=\frac{E}{\pi} \int_{\xi_{1}}^{\xi_{2}}(\mathbf{M}(\xi): \mathbf{E})^{2} \mathrm{~d} \xi=\frac{E}{\pi} \int_{\xi_{1}}^{\xi_{2}}\left(E_{1} \cos ^{2} \xi+E_{2} \sin ^{2} \xi\right)^{2} \mathrm{~d} \xi$

$\psi_{f}^{G S T}=\frac{E}{2}(\mathbf{H}: \mathbf{E})^{2}=\frac{2 E}{\pi^{2}}\left(\int_{\xi_{1}}^{\xi_{2}}\left(E_{1} \cos ^{2} \xi+E_{2} \sin ^{2} \xi\right) \mathrm{d} \xi\right)^{2}$

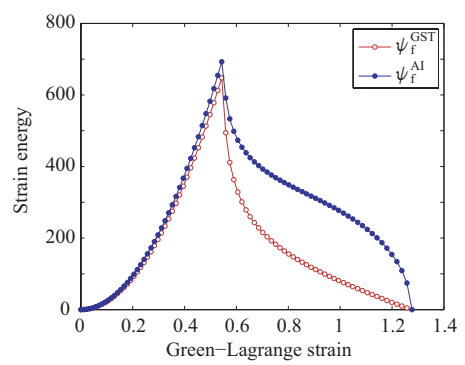

Figure 3: Comparison of the macroscopic strain energies of the fibrous tissue for the two homogenization methods with the proposed damage model for an increasing strain amplitude.

Besides, the components of tensor PK2 displayed on$$
\text { the }
$$

We can also observe a clear difference between the two homogenization methods in the concavity of the stress component in the least loaded direction. The softening part of the constitutive relation is much more anisotropic using the $A I$ model than using the $G S T$ model. The increase of the stress observed on $T_{f}^{A I}(22)$ 


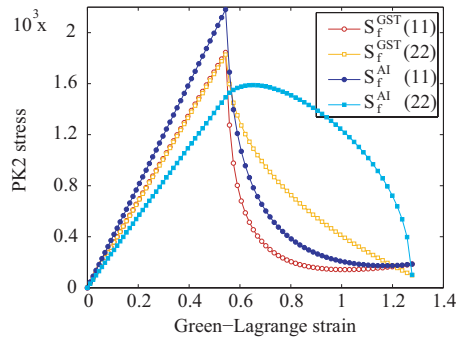

a)

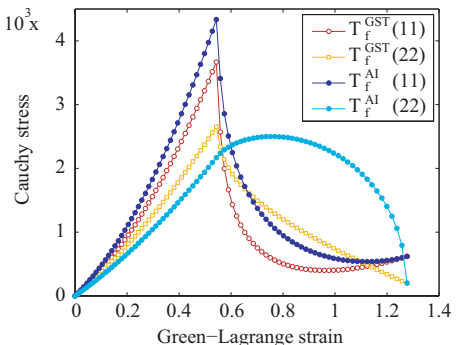

b)

Figure 4: Evolution of the tensor components of PK2 (a) and Cauchy (b) stresses as functions of the loading strain amplitude. The stress components do not revert back to 0 and start increasing after complete 413 rupture of the fibers because of the contribution of the matrix. just after the beginning of damage can be explained by the combination of an increasing applied strain and a relatively stable amount of load-carrying fibers in the 22 direction.

The homogenization methods have been compared on a theoretical point of view; let's now compare their behavior on an experimental case.

\section{Method to determine the model parameters using inflation test}

The identification method is based on the comparison ${ }^{429}$ of the simulated and experimental displacement fields of an inflated membrane. This section aims at describing the main steps of this procedure.

\subsection{Simulation of the experimental test}

The objective is to simulate the test as close as possi- ${ }_{435}$ ble to the real experimental conditions, by reproducing ${ }_{436}$ as well as possible the geometry and boundary condi- ${ }_{437}$ tions of the sample. To do so, considering global mea- ${ }_{438}$ surement (pressure and deflection of the pole) and ideal ${ }_{439}$ boundary conditions (corresponding to an axisymetric 440 configuration) is not satisfactory as they do not take ${ }_{441}$

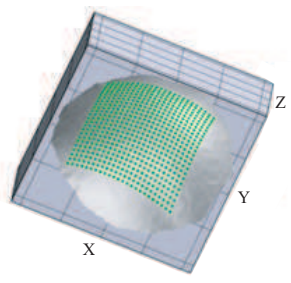

Figure 5: Nodes of the grid where the experimental displacement is caught. The simulation mesh is also defined on these nodes.

\section{.}

\section{mea} cant measure of the error for this is the most signifitical position of the $N$ nodes $i$ at each simulation step $j$, stored in $\mathbf{z}^{(j)}$, was used to determine the error $e$ between experimental and numerical position of the membrane: 

with $\mathrm{P}$ the number of steps in Abaqus, $\mathrm{N}$ the number ${ }_{478}$

$$
\begin{gathered}
e=\sum_{j=1}^{P} \sum_{i=1}^{N} \frac{\delta_{i}^{(j)}}{z_{p i}^{(j)}} \\
\delta_{i}^{(j)}=\left|z_{i}^{(j)}-z_{p i}^{(j)}\right|
\end{gathered}
$$
of nodes and $\delta_{i}^{(j)}$ the vertical gap between the experimental and numerical positions of node $i$ at step $j$. One issue here was that, as mentioned above, the correlation and simulation steps did not match. The measure of the gap between the simulated and experimental positions of a node was therefore not straightforward. We chose to vertically project the simulated position on the segment linking the previous and following experimental positions. $\delta_{i}^{(j)}$ was the gap between the simulated position $z_{i}^{(j)}$ and its projection on the experimental curve ${ }_{488}$ $z_{p i}^{(j)}$ (Fig.6). One may pay attention to the quantity of 489 correlation steps: the linear interpolation was satisfac- 490 tory as long as the correlation steps were frequent in the 491 non-linear areas (beginning of the curve).

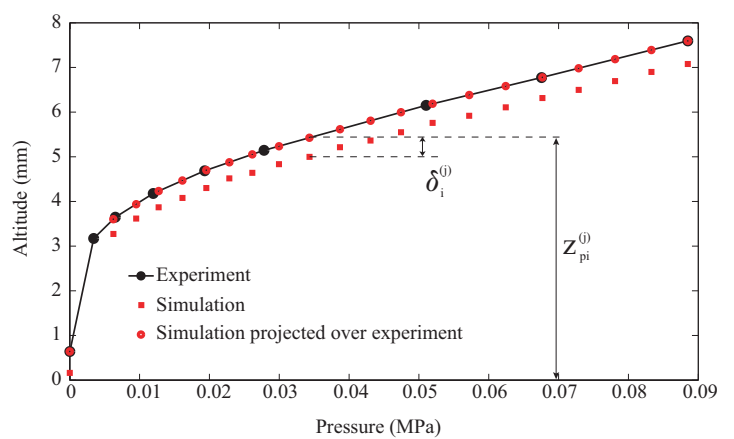

Figure 6: Measurement of the error in the vertical displacement used 503 for the identification procedure.

The algorithm of Levenberg-Marquardt (Levenberg, 1944; Marquardt, 1963) was chosen to ensure a good ${ }^{50}$ convergence of the minimization of the error and imple- 507 mented in Matlab. The Matlab routine wrote the succes- 508 sively required Abaqus input files, launched the Abaqus 509 simulations using the command function, and compiled 510 and ran the Fortran post-treatment files to get the node 511 displacements. The obtained simulated displacements 512 were read to build the Jacobian matrix and to further up- 513 date the material parameters and the regularization fac- 514 tor. Several initial guesses were tested to ensure that the 515 identified parameter corresponded to a global minimum. 516

In the case of the present damage model, the three pa- 517 rameters to identify were the fibers and the groundma- 518 trix Young's moduli, as well as the fibers ultimate strain. 519 conducted until the last image before the sample rupture, corresponding to a pressure $p_{\text {last }}$. We assumed then that the pressure increase $\Delta p$ between two images was constant at this stage, so that the ultimate pressure was known $\left(p_{\text {ult }}=p_{\text {last }}+\Delta p\right)$. The image and pressure ac-

As the contributions of both the matrix and the fibers are independent, the solution of the identification of the Young's moduli is not unique. An additional statement was necessary; in the present paper, we assumed that the matrix had a very small influence. Its modulus has been chosen to be about one thousand times smaller than the fibers modulus. Preliminary studies within this work led to choose a value of $0.01 \mathrm{MPa}$.

The determination of the fibers ultimate longitudinal strain was conducted using the ultimate pressure and deformation state of the material. As mentioned before, he matrix was much softer than the fibers. Therefore, a classical Finite Element simulation, without any improved tool to compute failure (such as X-FEM), lead a divergent result once the fibers break. SDIC was quisition frequency of $50 \mathrm{~Hz}$ is fast enough to ensure a small pressure increase between two images and therefore, a good estimation of the rupture pressure. The fiber ultimate strain corresponded to a divergent computation for this specific pressure.

\section{Application to human liver capsule}

The damage model and identification procedure presented in the previous sections were applied to human liver capsule. This tissue can indeed be considered as isotropic as confocal microscopy on the collagen fibers of the capsule did not reveal any preferred direction (Brunon et al, 2011). The experimental protocol has been presented in a previous paper (Brunon et al, 2011); the main features are recalled here.

\subsection{Experimental set-up}

Inflation tests were conducted on 15 samples of human liver capsule, all from the same liver. After being covered with a fine random pattern, the circular samples were fixed between two silicone flat seals $\left(\phi_{\text {int }}=25 \mathrm{~mm}\right.$, $\phi_{e x t}=30 \mathrm{~mm}$ ) on a PMMA plate (Fig.7). The circular shape of the samples was chosen so that the inflation test corresponded to rather equibiaxial tension. The capsule being translucent allowed a throughout lighting which prevented possible light reflections on the camera sensors and ensured a good SDIC. The capsule was inflated with air at a strain rate of approximately $10^{-2} \mathrm{~s}^{-1}$ before rupture. The deformation of the capsule was recorded by two digital DALSA cameras associated to two 35 
$\mathrm{mm}$ macro-lenses to have the appropriate size $\left(20 \times 20{ }_{541}\right.$ $\mathrm{mm}^{2}$ ) and depth of field (around $10 \mathrm{~mm}$ ). The pressure 542 was recorded using a 3-bar ENTRAN EPX-N1 pressure ${ }_{543}$ sensor (accuracy $\pm 1 \%$ ).
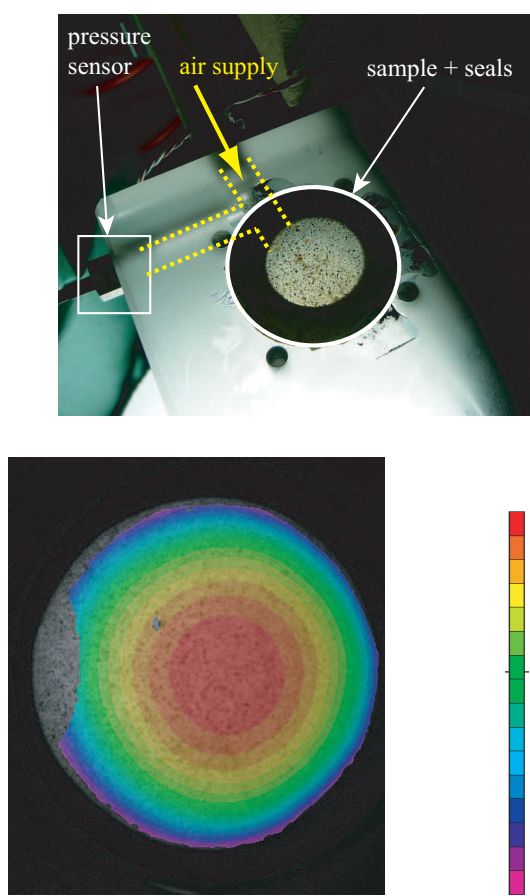

$8.9 \mathrm{~mm}$
Figure 7: Top: experimental device: view of the dedicated system to load the sample (without the clamp and screws) when illuminated. Bottom: Example of the vertical displacement field (in $\mathrm{mm}$ ) computed by stereocorrelation on the inflated capsule.

\subsection{Simulation of the test}

The method to simulate the test is described in 570 Sec.3.1 but some details specific to this application are 577 given here.

For the simulation, the thickness of the capsule 579 was assumed homogeneous throughout the liver. Its 580 value was set to $0.1 \mathrm{~mm}$, corresponding to a typical 581 mean value of measured thicknesses in previous studies 582 (Snedeker et al, 2005; Hollenstein et al, 2006; Brunon ${ }_{583}$ et al, 2010). The number of elements was fixed by the ${ }_{584}$ grid size $-0.5 \times 0.5 \mathrm{~mm}^{2}$ - of the SDIC. The number of ${ }_{585}$ elements depended on the sample and was around 500. 586

The reference - unloaded - state of the capsule was 587 defined after computing the position of the capsule by 588 SDIC. We set the reference state to be the first state (im- 589 age) with a constant curvature sign. This means that 590 there was no more wrinkling and that the displacements 591 further computed relative to this reference state would be consistent. The capsule was therefore slightly inflated at this reference state; the initial pressure was measured around $0.002 \mathrm{MPa}$, corresponding to less than $4 \%$ of the maximal measured pressure. The main difficulty as we will see in the next section is the control of the clamping conditions. In order to have a good modeling of the real boundary conditions, the SDIC-computed displacements have been used to set the boundary conditions of the model.

The simulation was conducted using an explicit procedure. This resulted in vibrations of the capsule if the simulation speed was the real one. Therefore the simulated test was five times slower than the experimental test; this ensured a good representation of the beginning of the deflection curve and a good convergence of the identification algorithm.

To ease the convergence of the identification, the optimization procedure was conducted in two steps: we first minimized the error on the pole of the sphere vertical position ; this gave a first estimation of the parameters. Then we adjusted these previously determined parameters using the error on all the nodes of the capsule. This method revealed that the position of the pole was a rich enough information to identify the parameters of the damage law, as the optimization of the parameters during the second phase lead to less than $5 \%$ of variation of the identified parameters provided the experimental boundary conditions are correctly modeled.

\subsection{Results}

As mentioned in (Brunon et al, 2011), the experimental strain field did not correspond to an ideal axisymetric inflation test. Due to their softness, the silicone seals wrinkled while being compressed and made the clamping not flat. Also, a few samples experienced slippage. This shows the need for full-field measurements to ensure the correspondence between the experimental test and the simulated one.

Fig. 8 shows the result of the identification of the fibers Young's modulus. The matrix modulus was chosen to be $0.01 \mathrm{MPa}$ as preliminary studies showed that the fibers modulus would be in the order of $10 \mathrm{MPa}$. This lead to a value of $19 \pm 6 \mathrm{MPa}$ for the fibers elasticity (Tab.1). This value corresponds to an error between the experimental and simulated displacements of $4 \%$ when averaged over all the nodes and steps. It is obvious here that the linear assumption is not sufficient as it is too stiff at the beginning and not enough for larger strains. No significant difference was detected between the $A I$ and the GST versions of the model; this is due to the assumption of linear behavior for the fiber - or for 


\begin{tabular}{|c|c|c|c|}
\hline Sample nb & E (MPa) & $\varepsilon_{r}^{A I}(-)$ & $\varepsilon_{r}^{G S T}(-)$ \\
\hline 1 & 10.0 & 0.435 & 0.345 \\
2 & 20.0 & 0.290 & 0.275 \\
3 & 19.6 & 0.305 & 0.275 \\
6 & 30.5 & - & - \\
7 & 24.0 & 0.390 & 0.345 \\
8 & 19.8 & - & - \\
9 & 20.0 & 0.355 & 0.340 \\
10 & 11.3 & 0.290 & 0.275 \\
11 & 25.3 & - & - \\
12 & 14.9 & 0.345 & 0.335 \\
13 & 25.4 & 0.315 & 0.300 \\
14 & 14.0 & 0.365 & 0.360 \\
15 & 12.1 & - & - \\
\hline
\end{tabular}

Table 1: Identified values of the fibers Young modulus and determined values of their ultimate strain, for both $A I$ and GST homogenization method. Cells exhibiting "-." correspond to samples that experienced slippage and did not break. Only the values of Young's modulus obtained with the $A I$ method are displayed as they were the same as the ones obtained with the GST method.

the whole fibrous tissue - which makes the two models equivalent without any damage.

For the identification of the ultimate strain $\epsilon_{r}$, the ultimate pressure was set to $105 \%$ of the pressure corresponding to the last image before rupture, as explained in Sec.3.2. A mean value of $33 \pm 4 \%$ is obtained. The two versions of the model give approximately the same results in terms of ultimate strain of the fiber.

Fig.9 shows the failure surface obtained with both model. With ideal boundary conditions, the capsule sample being circular would lead to an equal loading on all the fibers and a brutal rupture of all the fibers at the same step. Using the experimental boundary conditions leads to a non-equibiaxial loading and a more localized rupture, especially with the $G S T$ version model. We can see the damage and the strain concentration in several elements (light to dark blue). The $A I$ version of the model leads to a faster increase of damage in all the elements: the loading is indeed much more biaxial with this version once the damage occurs than with the GST version, as we can see on the Fig.4; the stress in the less loaded direction is still quite high while it drops really fast in the $G S T$ version.
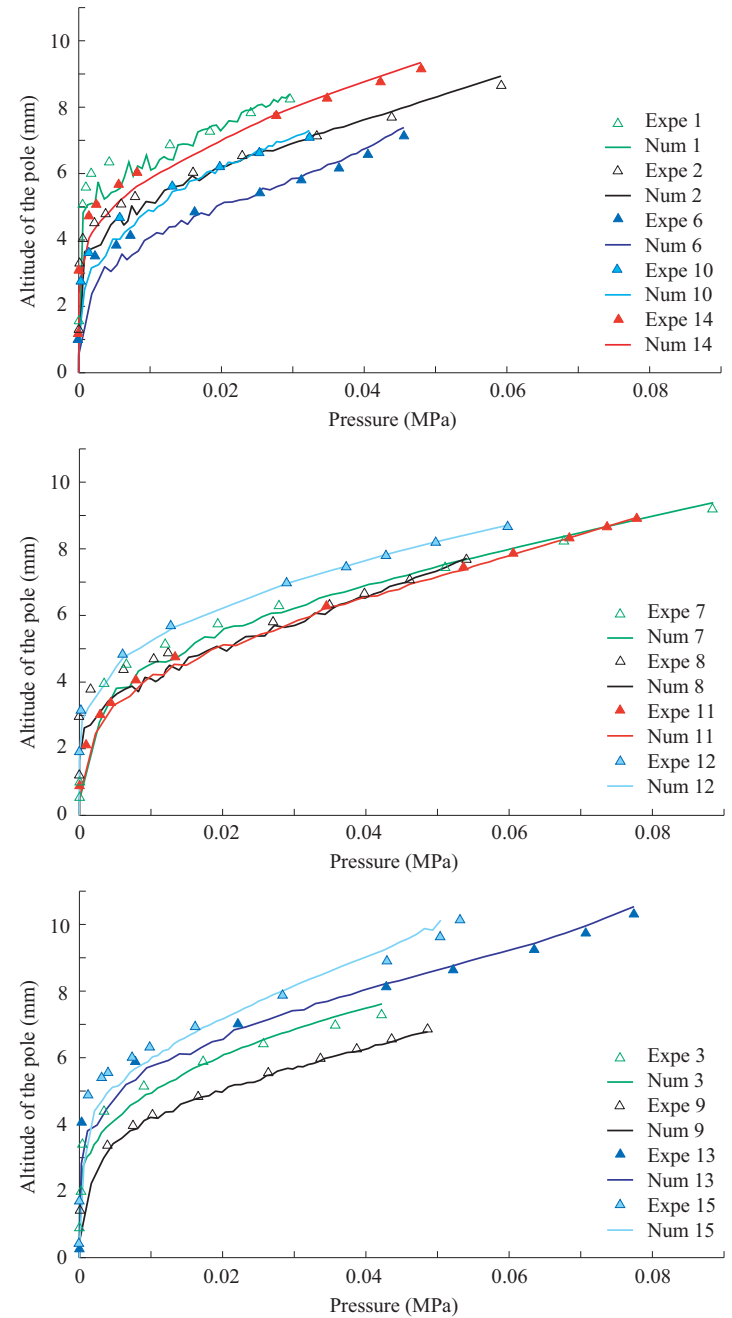

Figure 8: Identification of the GST version of the damage model for human hepatic capsule. Expe and Num are experimental data and simulated data respectively. 


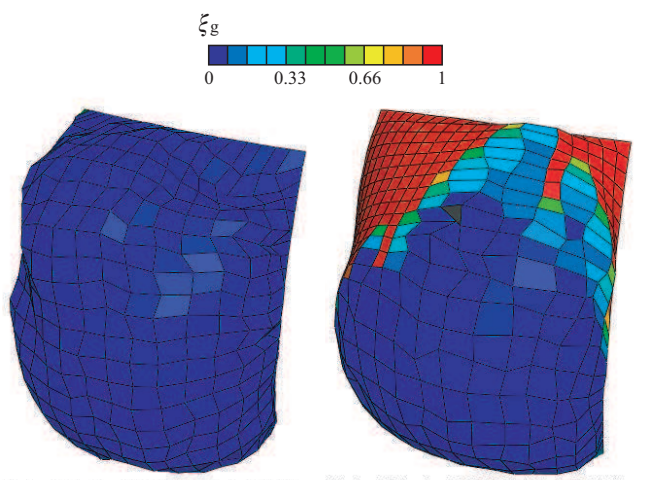

Figure 9: Comparison of the failure surface obtained with both homogenization methods (left: $A I$, right:GST). $\xi_{g}=\frac{2}{\pi}\left(\xi_{2}-\xi_{1}\right)$ so $\xi_{g}=1$ (red) with no damage and decreases to 0 (blue) when dam- 664 age increases.

This paper presents the comparison between two ho- 670 mogenization methods on the theoretical and experi- 671 mental point of views. It gives a first estimation of the 672 material properties of the human liver capsule through a 673 realistic loading. Biaxial tension is close to what the 674 liver surface experiences on one region if it is com- 675 pressed on the other side (which is typical of crash load- 676 ing situations). However more samples from more livers 677 should be tested to give an actual value for the identified 678 parameters.

The damage model described in this paper is adapted 680 to the human liver capsule as its fiber angular distri- 681 bution is homogeneous (Brunon et al, 2011). It al- 682 lows a satisfactory description of this tissue within a 683 small number of parameters, which makes the identi- 684 fication procedure rather robust. The assumption of lin- 685 ear elasticity for the elastic part is however not correct 686 even if the strain only reaches $30 \%$; as seen in (Brunon 687 et al, 2011), a non-linear law could represent more accu- 688 rately the increase of stiffness as the strain increases. Of 689 course more sophisticated damage models available in 690 the literature would better describe some physical fea- 691 tures of the capsule, such as its non-linearity, its vis- 692 coelasticity (not mentioned in this paper but existing as 693 in most of the biological tissues), the fiber crimp or the 694 damage nature of the fibers themselves. However, such 695 features were not in the scope of this study which first, 696 was focused on the homogenization methods and sec- 697 ond, aimed at describing the tissue with very few pa- 698 rameters to allow a robust identification. The construc- 699 tion of the model in its two versions showed that the ho- 700 mogenization methods differ significantly once damage 701 starts. Also it revealed a non-physical behavior for the second Piola-Kirchhoff tensor when the GST method is applied to an isotropic planar tissue loaded with nonequibiaxial tension.

The identified parameters (fibers Young's modulus and ultimate longitudinal strain) are a preliminary estimation as all the samples come from the same liver. The groundmatrix elasticity modulus, which is a parameter of the complete model, cannot be identified using this protocol for two reasons: the parallel contribution of both the fibers and the groundmatrix leads to a nonunique solution for the identification of their Young's moduli; the groundmatrix contribution to the overall energy and stress is very low in a connective tissue, so that the experimental noise prevents a robust identification of its elasticity. Therefore the matrix elasticity modulus was set up to an arbitrary low value. Comparing the fibers Young's modulus obtained here to values from the literature would require better knowledge on the tissue microstructure. The capsule is mainly made of collagen fibers of type I and III. The value of $19 \mathrm{MPa}$ is rather low compared to those from the literature that range for collagen of type I from 0.4 to $3 \mathrm{GPa}$ (Fung, 1993; Sasaki and Odajima, 1996; Carlisle et al, 2010). Only one paper has comparable values (Lopez-Garcia et al, 2010). A quantification of the microstructure would be necessary to explain this discrepancy; previous studies showed indeed the strong influence of structure over stiffness of the collagen (Gautieri et al, 2011).

On the other hand, the ultimate strain determined using this damage model can be compared to the literature as it does not depend on the quantity of fibers in each direction. The value of $33 \pm 4 \%$ is in the range of the the one assessed in (Carlisle et al, 2010).

The main feature of the identification method is the simulation of the test using experimental boundary conditions. One of the main issues when testing soft tissues is to ensure the repeatability of the boundary conditions from one sample to the other. The need for special clamping technics, that do not damage the sample and prevent any sliding implies that the boundary conditions are not perfectly controlled, especially with such a thin tissue. Using full-field measurement with high quality images allows determining the actual strain field on the sample rather accurately. In our case, several samples experienced slippage or wrinkled stress free states but these experimental characteristics are caught by the SDIC and included in the simulation. Once automatized, the identification procedure can therefore take into account the variability of the experimental conditions, to improve the material parameters determination. Simulating the tests using the experimental boundary 
conditions allows the comparison between the two ver- 751 sions of the damage model. In the inflation test case, 752 the tissue is loaded with pressure; this emphasizes the ${ }^{753}$ difference in damage progression between the two ho- ${ }_{755}^{754}$ mogenization methods.

The main issue of the experimental protocol is that 75 the membrane failure occurs rather rapidly, which does ${ }_{758}^{758}$ not allow the control of the damage evolution. This 760 is due to two main reasons. First, the capsule was in- 761 flated with air, which is compressible. When the failure ${ }^{76}$ started, the compressed air was suddenly released and ${ }_{764}^{763}$ made the failure grow almost instantly. This could be 765 improved by applying the load using water instead of 766 air: as water is incompressible, the membrane displace- ${ }^{767}$ ment during inflation would be more directly controlled, ${ }^{768}$ especially when failure occurs. Second, the circular 770 shape of the clamping made the load almost equibiaxial. ${ }^{77}$ As the membrane was initially isotropic, this lead to an ${ }^{772}$ equal loading of all the fibers, as shown in the model de- ${ }_{774}^{773}$ scription. An elliptic instead of circular clamping could 775 allow a slower increase of the damage into the tissue ${ }^{776}$ and therefore, it could be caught by the SDIC system. ${ }_{778}^{777}$

The identification method presented here can be 779 adapted to any soft tissue membrane. Existing or user- 780 defined constitutive laws can be accurately identified as ${ }^{781}$ the experimental conditions are correctly simulated. For ${ }_{783}^{782}$ this particular application, future work would consist in 784 implementing non-linear potential for the fibers in the 785 damage model and modifying the experimental set-up ${ }^{78}$ to ensure a better control of the failure (shape of the ${ }^{78}$ clamp, loading with an incompressible fluid). An in- 789 teresting prospect would also be to identify both con- ${ }^{790}$ tributions of the fibers and the groundmatrix; this could be achieved by treating the tissue with collagenase to ${ }_{793}^{792}$ destroy the collagen fibers and characterize the ground- 794 matrix alone, as done in (Rausch et al, 2012). More advanced identification methods could also be adapted to this model such as the virtual field method - already used for a similar protocol in (Kim et al, 2011) - or the 799 Integrated Mechanical Image Correlation (I-MIC, see ${ }^{800}$ (Réthoré, 2010)). Such methods improve the noise sensitivity of the optimization procedure.

Acknowledgement 1. The authors would like to thank the Region Rhone-Alpes for its financial support as well as Adrien Charmetant for his technical support .

\section{References}

Ateshian GA (2007) Anisotropy of fibrous tissues in relation to the distribution of tensed and buckled fibers. Journal of biomechanical engineering 129:240-249
Balzani D, Schröder J, Gross D (2006) Simulation of discontinuous damage incorporating residual stresses in circumferentially overstretched atherosclerotic arteries. Acta Biomateriala 2(6):609-618

Bel-Brunon A, Coret M, Bruyère-Garnier K, Combescure A (2012) Comparaison of two homogenization methods using a damage model for a fibrous membrane, based on the fibers' fracture process at the microscale. European Journal of Mechanics - A/Solids 39:1-10

Bischoff J, Drexler E, Slifka A, McCowan C (2009) Quantifying nonlinear anisotropic elastic material properties of biological tissue by use of membrane inflation. Computer Methods in Biomechanics and Biomedical Engineering 12(3):353-369

Boyce B, Grazier J, Jones R, Nguyen T (2008) Full-field deformation of bovine cornea under constrained inflation conditions. Biomaterials 29(28):3896-3904

Brunon A, Bruyère-Garnier K, Coret M (2010) Mechanical characterization of liver capsule through uniaxial quasi-static tensile tests until failure. Journal of Biomechanics 43(11):2221-2227

Brunon A, Bruyère-Garnier K, Coret M (2011) Characterization of the nonlinear behaviour and the failure of human liver capsule through inflation tests. Journal of the Mechanical Behavior of Biomedical Materials 4(8):1572-1581

Cacho F, Elbischger PJ, Rodriguez JF, Doblare M, Holzapfel GA (2007) A constitutive model for fibrous tissues considering collagen fiber crimp. International Journal of Non-Linear Mechanics 42(2):391-402

Calvo B, Pena E, Martinez MA, Doblare M (2006) An uncoupled directional damage model for fibred biological soft tissues. Formulation and computational aspects. International Journal for Numerical Methods in Engineering 06:1-30

Carlisle C, Coulais C, Guthold M (2010) The mechanical stress-strain properties of single electrospun collagen type I nanofibers. Acta Biomaterialia 6(8):2997-3003

Cortes DH, Lake SP, Kadlowec JA, Soslowsky LJ, Elliott DM (2010) Characterizing the mechanical contribution of fiber angular distribution in connective tissue: comparison of two modeling approaches. Biomechanics and Modeling in Mechanobiology 9:651658

Decraemer WF, Maes M, Vanhuyse VJ (1980) An elastic stress-strain relation for soft biological tissues based on a structural model. Journal of Biomechanics 13:463-468

Fung Y (1993) Biomechanics: mechanical properties of living tissues, vol 12. Springer

Gasser T, Holzapfel G (2002) Modeling the propagation of arterial dissection. European Journal of Mechanics-A/Solids 25(4):617633

Gasser T, Ogden R, Holzapfel G (2006) Hyperelastic modelling of arterial layers with distributed collagen fibre orientations. Journal of the royal society interface 3(6): 15

Gautieri A, Vesentini S, Redaelli A, Buehler M (2011) Hierarchical structure and nanomechanics of collagen microfibrils from the atomistic scale up. Nano Letters 11(2):757-766

Gleason RLJR, Humphrey D (2005) A 2D constrained mixture model for arterial adaptations to large changes in flow, pressure and axial stretch. Mathematical Medecine and Biology 22:347-396

Hill MR, Duan X, Gibson GA, Watkins S, Robertson AM (2012) A theoretical and non-destructive experimental approach for direct inclusion of measured collagen orientation and recruitment into mechanical models of the artery wall. Journal of Biomechanics 45:762-71

Hollenstein M, Nava A, Valtorta D, Snedeker J, Mazza E (2006) Mechanical Characterization of the Liver Capsule and Parenchyma. Lecture notes in computer science 4072:150-158

Holzapfel GA, Ogden RW (2010) Constitutive modelling of arteries. Proceedings of the Royal Society A: Mathematical, Physical and 
Engineering Science 446(2118):1551-1597

Hurschler C, Loitz-Ramage B, Vanderby JR (1997) A structurally 882 based stress-stretch relationship for tendon and ligament. Journal 883 of biomechanical engineering 119:392-399

Johannknecht R, Jerrams S (1999) The need for equi-biaxial testing 885 to determine elastomeric material properties. In: Proceedings of 886 the First European Conference on Constitutive Models for Rubber, 887 Vienna, Austria, 9-10 September 1999, Taylor \& Francis, 73-76 888

Kim J, Avril S, Duprey A, Favre J (2011) Experimental characterization of rupture in human aortic aneurysms using a full-field measurement technique. Biomechanics and Modeling in Mechanobiology 11(6):841-853

Lanir Y (1983) Constitutive equations for fibrous connective tissues. Journal of Biomechanics 16:1-12

Levenberg K (1944) A method for the solution of certain problems in least squares. Quarterly of Applied Mathematics 2:164-168

Liao J, Yang L, Grashow J, Sacks M (2005). Molecular orientation of collagen in intact planar connective tissues under biaxial stretch. Acta Biomaterialia 1:45-54

Limbert G, Middleton J (2004) A transversely isotropic viscohyperelastic material:: Application to the modeling of biological soft connective tissues. International journal of solids and structures 41(15):4237-4260

Lopez-Garcia M, Beebe D, Crone W (2010) Young's modulus of collagen at slow displacement rates. Bio-Medical Materials and Engineering 20(6):361-369

Marquardt D (1963) An algorithm for least-squares estimation of nonlinear parameters. Journal of the society for Industrial and Applied Mathematics 11(2):431-441

Marra S, Kennedy F, Kinkaid J, Fillinger M (2006) Elastic and rupture properties of porcine aortic tissue measured using inflation testing. Cardiovascular Engineering 6(4):123-131

Mohan D, Melvin J (1983) Failure properties of passive human aortic tissue. ii-biaxial tension tests. Journal of Biomechanics 16(1):3137

Orberg JW, Klein L, Hiltner A (1982) Scanning electron microscopy of collagen fibers in intestine. Connective tissue research 9(3):187193

Rausch S, Pack O, Martin C, Uhlig S, Wall W (2012) Development of a constituent-based material model for soft biological tissue using the example of lung parenchyma. Journal of the Mechanical Behavior of Biomedical Materials, submitted.

Réthoré J (2010) A fully integrated noise robust strategy for the identification of constitutive laws from digital images. International Journal for Numerical Methods in Engineering 84 (6):631-660

Rodríguez JF, Cacho F, Bea JA, Doblaré M (2006) A stochasticstructurally based three dimensional finite-strain damage model for fibrous soft tissue. Journal of the Mechanics and Physics of Solids 54(4):864-886

Sacks M, Gloeckner D (1999) Quantification of the fiber architecture and biaxial mechanical behavior of porcine intestinal submucosa. Journal of biomedical materials research 46(1):1-10

Sasaki N, Odajima S (1996) Elongation mechanism of collagen fibrils and force-strain relations of tendon at each level of structural hierarchy. Journal of biomechanics 29(9):1131-1136

Snedeker J, Niederer P, Schmidlin F, Farshad M, Demetropoulos C, Lee J, Yang K (2005) Strain-rate dependent material properties of the porcine and human kidney capsule. Journal of Biomechanics 38(5):1011-1021

Sutton M, Orteu J, Schreier H (2009) Image correlation for shape, motion and deformation measurements: basic concepts, theory and applications. Springer Verlag.

Svensson RB, Hassenkam T, Hansen P, Magnusson SP (2010) Viscoelastic behavior of discrete human collagen fibrils. Journal of the Mechanical Behavior of Biomedical Materials 3:112-115
Tinkoff G, Esposito T, Reed J, Kilgo P, Fildes J, Pasquale M, Meredith J (2008) American Association for the Surgery of Trauma Organ Injury Scale I: spleen, liver, and kidney, validation based on the National Trauma Data Bank. Journal of the American College of Surgeons 207(5):646-655

Viidik A (1972) Simultaneous mechanical and light microscopic studies of collagen fibers. Zeitschrift für Anatomie und Entwicklungsgeschichte 136(2):204-212 